\title{
Strong coupling beyond the light-line
}

\section{(Supplementary Information)}

\author{
Kishan S. Menghrajani* and William L. Barnes* \\ Department of Physics and Astronomy, University of Exeter, Exeter, \\ EX4 4QL, United Kingdom \\ E-mail: k.s.menghrajani@exeter.ac.uk; w.I.barnes@exeter.ac.uk
}

\section{Contents}

1 Grating morphology

2 Thickness dependence of Rabi-splitting with the $\mathrm{TM}_{-1}$ mode

3 Field profiles of the $\mathrm{TE}_{0}, \mathrm{TM}_{0}$, and $\mathrm{TM}_{-1}$ modes

4 Effect of superstrate index on dispersion of coupled surface plasmon mode S7

5 Calculated dispersion of continuous and discontinuous (stripe array) one dimensional gold grating structures

6 Dispersion of DBR cavity modes for zero oscillator strength

$7 \quad$ Line spectra

8 Field profiles of the $\mathrm{TE}_{0}, \mathrm{TM}_{0}$ modes at higher $k_{x}$ 
9 Oscillator models for one molecular resonance and several photonic resonances

\section{Grating morphology}

Our 1-D gratings were made using electron beam lithography of evaporated $30 \mathrm{~nm}$ gold films on $\mathrm{CaF}_{2}$ substrates. An example of the grating structures we used in this work is shown in the atomic force microscopy (AFM) picture shown in figure S1. In this case the nominal grating period was $4.7 \mu \mathrm{m}$ and the slot width and height were $\sim 1.0 \mu \mathrm{m}$, and $\sim 30 \mathrm{~nm}$ respectively.

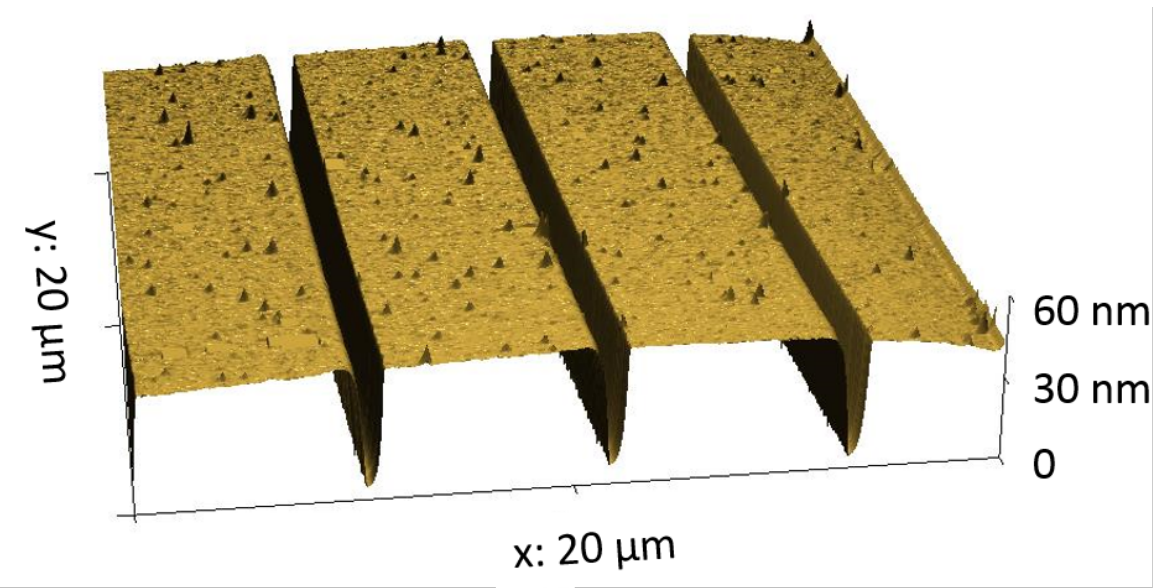

Figure S1: AFM image of a $\sim 30 \mathrm{~nm}$ thick gold grating on a $\mathrm{CaF}_{2}$ substrate. 


\section{Thickness dependence of Rabi-splitting with the $\mathrm{TM}_{-1}$}

\section{mode}

Here we investigate the extent of the Rabi-splitting involving the coupled plasmon $\left(\mathrm{TM}_{-1}\right)$ mode, as a function of the thickness of the cavity. We used numerical simulations based on the Fresnel approach. In figure S2 three examples of the calculated dispersion are shown, in figure S3 the thickness dependence of the Rabi splitting is shown, calculated using data such as those shown in figure S2.
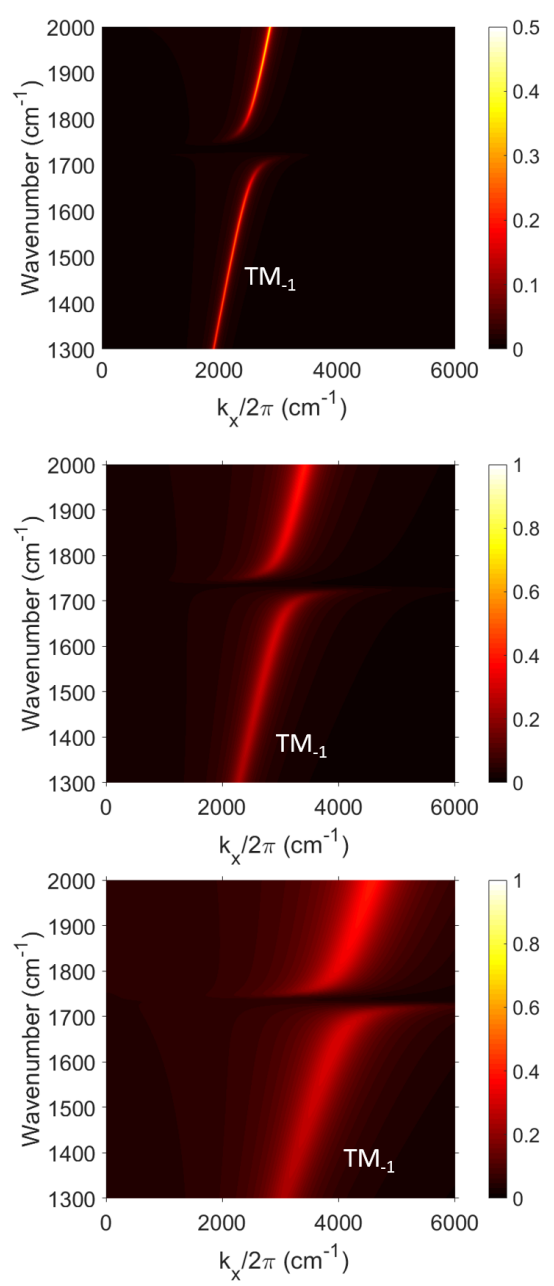

Figure S2: Dispersion of coupled plasmon modes for three different cavity (PMMA) thicknesses. Data shown were calculated numerically using a Fresnel-based approach. The cavity thicknesses are: (upper) $1 \mu \mathrm{m}$; (middle) $100 \mathrm{~nm}$; and (lower) $30 \mathrm{~nm}$. The absolute value of the p-polarised amplitude transmission coefficient is shown as a function of frequency and wavevector. 


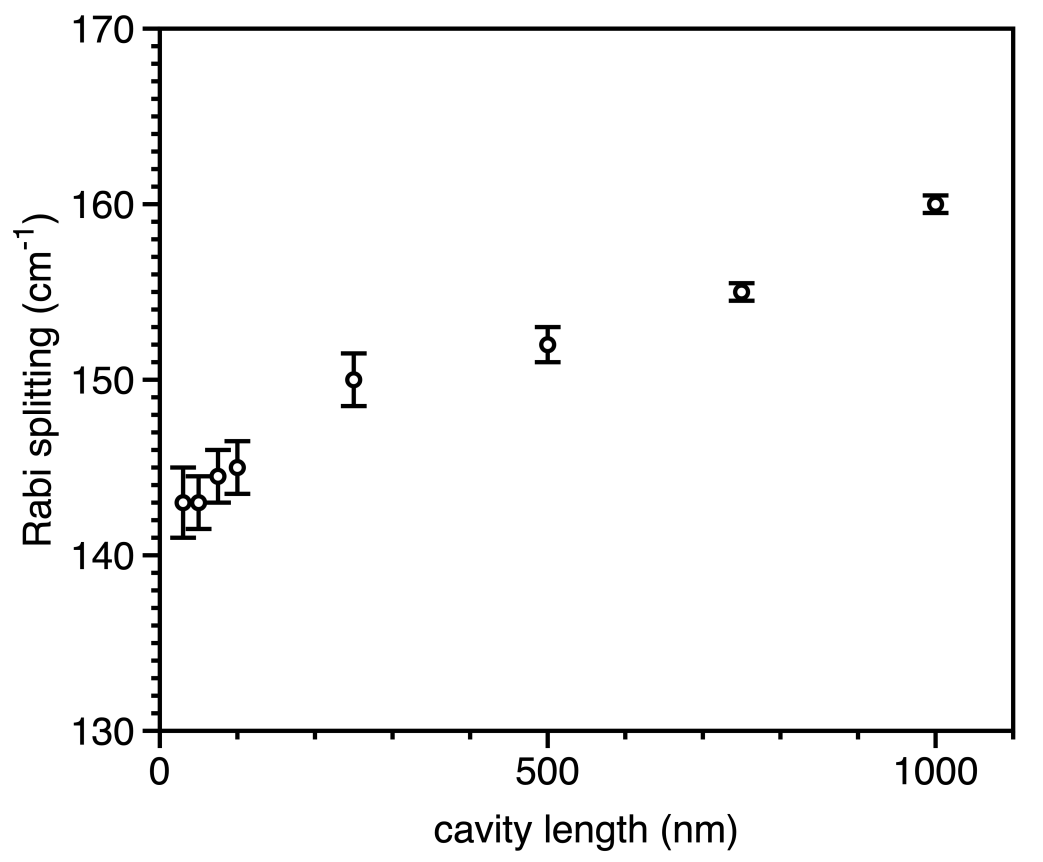

Figure S3: Rabi splitting as a function of cavity thickness. The error bars represent the confidence range in estimating the Rabi splitting from Fresnel-derived dispersion data 


\section{Field profiles of the $\mathrm{TE}_{0}, \mathrm{TM}_{0}$, and $\mathrm{TM}_{-1}$ modes}

The data produced here are again from simulations using the Fresnel approach. The field profiles were calculated for various combinations of frequency and wavevector. To aid association of these $\left(\omega, k_{x}\right)$ values with the different modes we first show dispersion plots for TE (figure S4a) and TM (figure S4b) polarisations. The absolute value of the s- and ppolarised amplitude transmission coefficients are shown, as appropriate, as a function of frequency and wavevector.
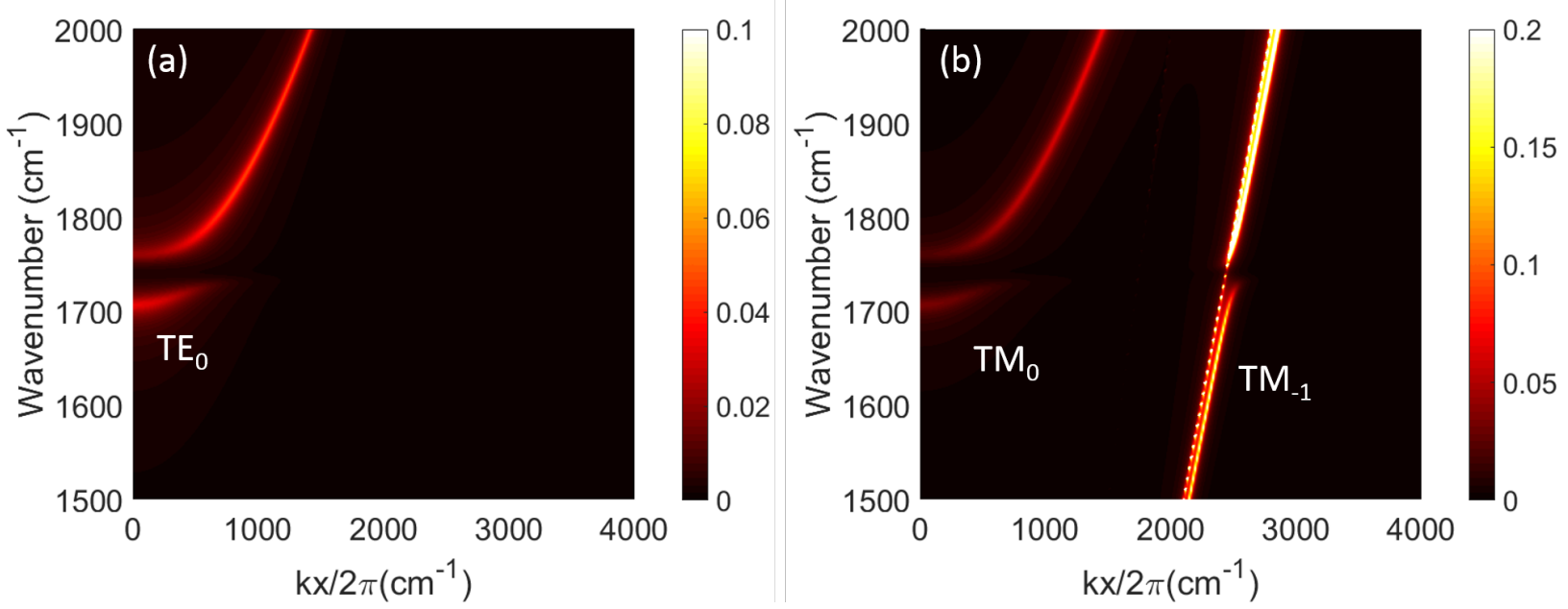

Figure S4: Dispersion plot The absolute value of the complex TM-polarised and TEpolarised amplitude transmission coefficients in the presence of the molecular resonance (vibrational mode) is shown in (a) and (b) respectively, as a function of frequency (wavenumber) and in-plane wavevector.

The field profiles for the $\mathrm{TE}_{0}$, for the $\mathrm{TM}_{0}$ and for the $\mathrm{TM}_{-1}$ were calculated for the lower polariton at a frequency of $1718 \mathrm{~cm}^{-1}$ and for the upper polariton at a frequency of $1772 \mathrm{~cm}^{-1}$. For the $\mathrm{TE}_{0}$ and $\mathrm{TM}_{0}$ field profile calculations the in-plane wavevector $\left(k_{x} / 2 \pi\right)$ was taken to be $382 \mathrm{~cm}^{-1}$, for the $\mathrm{TM}_{-1}$ mode a value of $2500 \mathrm{~cm}^{-1}$ was chosen for the in-plane wavevector. 

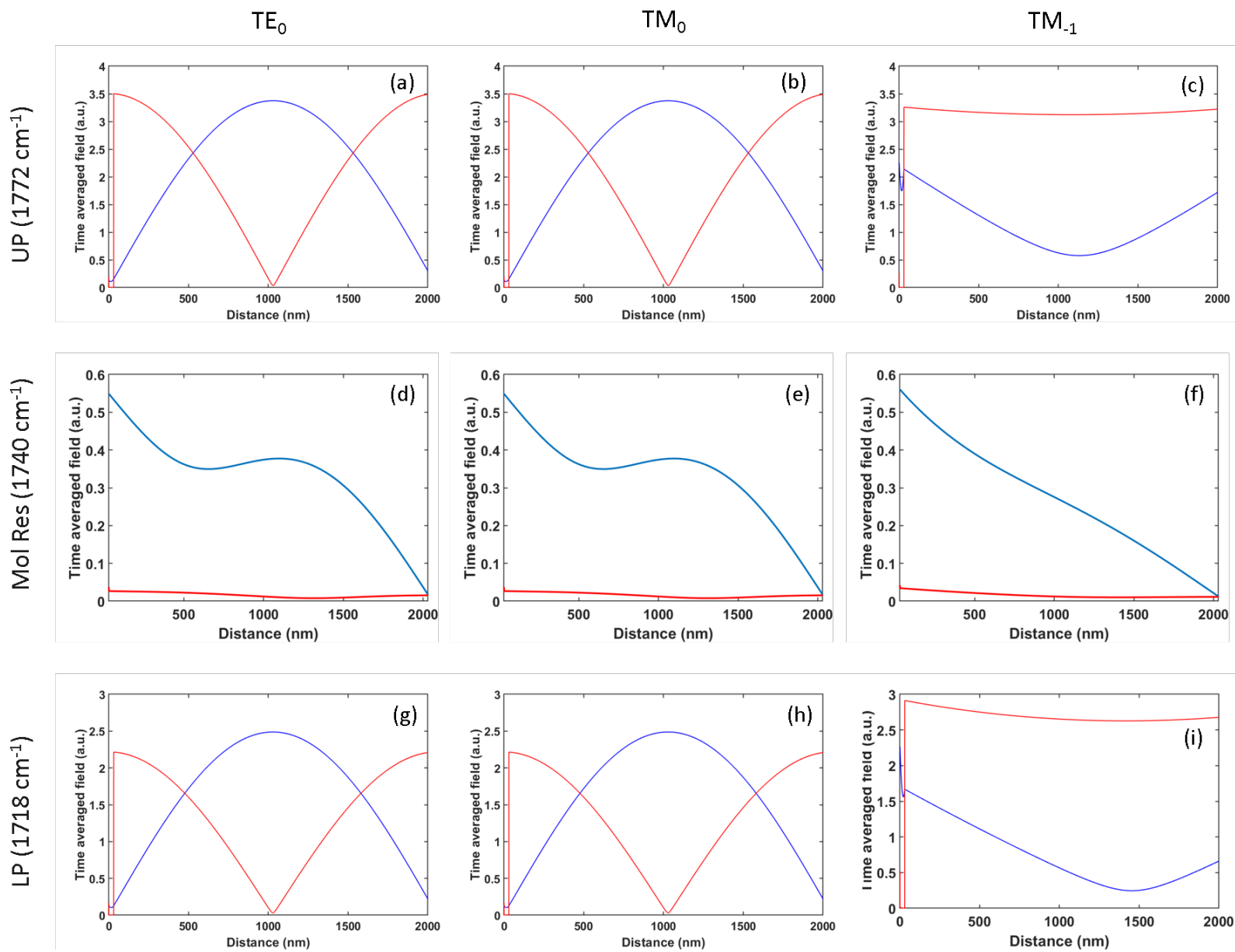

Figure S5: Field profiles. The plots show the time averaged electric fields. Left column: (a), (d) and (g) are for the $\mathrm{TE}_{0}$ mode, Middle column: (b), (e) and (h) for the $\mathrm{TM}_{0}$ mode, Right column: (c), (f) and (i) are for the $\mathrm{TM}_{-1}$ mode. Two field components are shown, in blue is the $\mathrm{E}_{x}$ (in-plane) component, in red is the $\mathrm{E}_{z}$ (out-of-plane) component. Top row: Upper polariton field profiles, calculated at a frequency of $1718 \mathrm{~cm}^{-1}$, and for the following in-plane wavevector values: $\mathrm{TM}_{0}$ and $\mathrm{TE}_{0}$ mode at $\sim 382 \mathrm{~cm}^{-1}, \mathrm{TM}_{-1}$ mode at $\sim 2500$ $\mathrm{cm}^{-1}$. Middle row: Field profiles calculated at the molecular resonance frequency of 1740 $\mathrm{cm}^{-1}$, and for the following in-plane wavevector values: $\mathrm{TM}_{0}$ and $\mathrm{TE}_{0}$ mode at $\sim 19 \mathrm{~cm}^{-1}$, $\mathrm{TM}_{-1}$ mode at $\sim 2530 \mathrm{~cm}^{-1}$. Bottom row: Lower polariton field profiles, calculated at a frequency of $1772 \mathrm{~cm}^{-1}$, and for the following in-plane wavevector values: $\mathrm{TM}_{0}$ and $\mathrm{TE}_{0}$ mode at $\sim 382 \mathrm{~cm}^{-1}, \mathrm{TM}_{-1}$ mode at $\sim 2500 \mathrm{~cm}^{-1}$. 


\section{Effect of superstrate index on dispersion of coupled surface plasmon mode}

Here we re-calculate the dispersion plots shown in the main manuscript in figure 1 (panels d-i), using the Fresnel approach. The difference here is that we have changed the superstrate index from the value of $n=10$ used in the main manuscript to the value of $n=1$ (as in the

experiments). For $n=10$ (figure 1, main manuscript) there is a sharp mode visible for the thin cavity that follows the air light-line, this is the surface plasmon mode on the top metal surface. This top surface plasmon is a confusing extra mode when trying to understand what happens to the coupled plasmon modes as the cavity gets thinner. That is not to say that the surface plasmon mode on the top surface should be ignored in a full analysis, simply that for the present purpose it is confusing and need not be considered here. Note that here for figure S6 and S7, the structures considered are as for figure 1, i.e: left-hand column, planar metal film; central column, planar metal cavity; right-hand column, planar metal cavity below cut-off. 
(a)

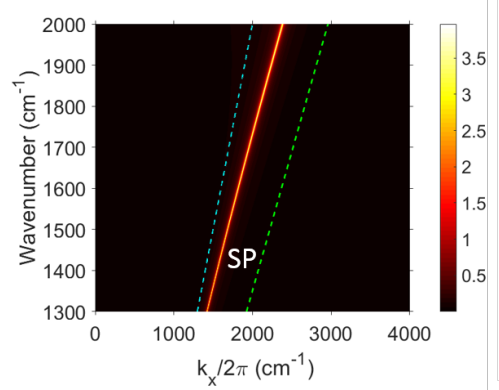

(b)

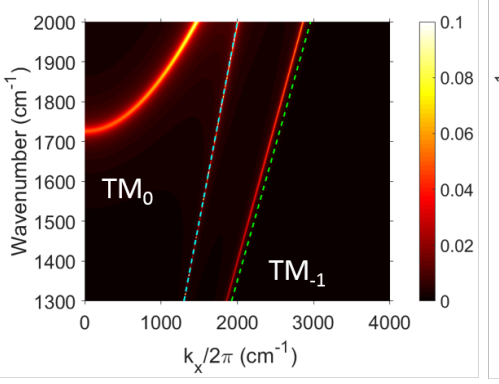

(c)

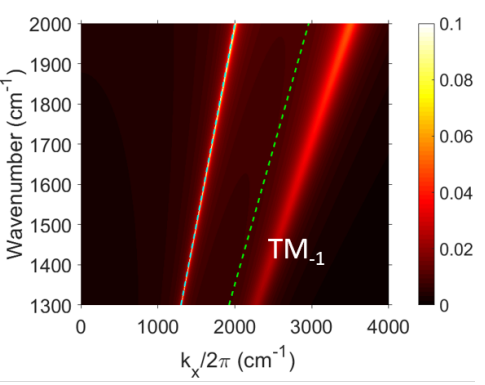

Figure S6: Dispersion of modes at $n=1.0$ and with oscillator strength turned off. Left (a) is the dispersion for a planar metal film, showing the surface plasmon (SP) mode of a single planar $30 \mathrm{~nm}$ gold metal film overlain with a $2 \mu \mathrm{m}$ polymer PMMA film on a $\mathrm{CaF}_{2}$ substrate; Middle (b) is for a cavity near cutoff of a $2 \mu \mathrm{m}$ thick film of PMMA sandwiched between two $30 \mathrm{~nm}$ thick planar gold mirrors on a $\mathrm{CaF}_{2}$ substrate, Right (c) A cavity well-below the usual cut-off of a $100 \mathrm{~nm}$ thick PMMA film sandwiched between two $30 \mathrm{~nm}$ thick planar gold mirrors on $\mathrm{a}_{\mathrm{CaF}}$ substrate. Notice that in addition to the $\mathrm{TM}_{-1}$ coupled plasmon mode there is also a surface plasmon mode at the gold/air interface )the superstrate modelled here - air - has a refractive index of 1 . This produces the gold/air surface plasmon shown that appears near the air light-line. In all three panels seen here the oscillator strength of the vibrational resonance has been set to zero. Dispersion plot based on Fresnel-type calculations. The absolute value of the TM polarised Fresnel coefficient is shown as a function of frequency (wavenumber) and in-plane wavevector.

(a)

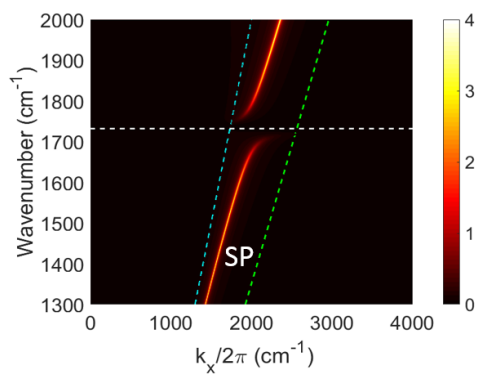

(b)

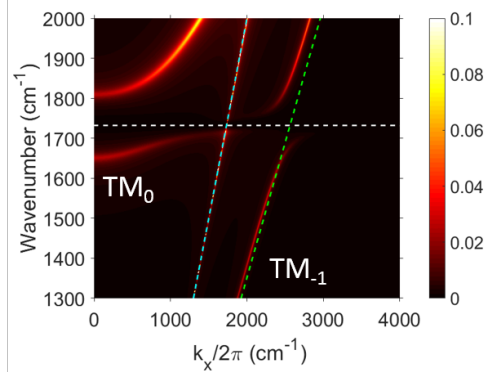

(c)

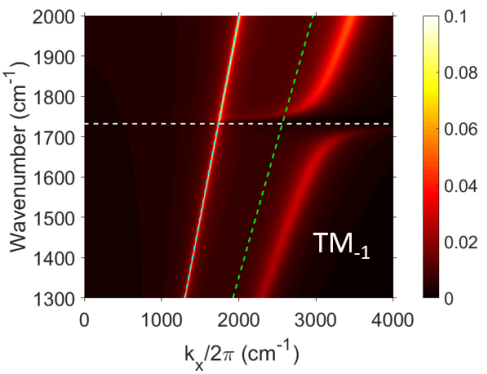

Figure S7: As figure S6, dispersion of modes at $n=1.0$ and with oscillator strength turned on. Dispersion plot based on Fresnel-type calculations. The absolute value of the TM polarised Fresnel coefficient is shown as a function of frequency (wavenumber) and in-plane wavevector. 


\section{Calculated dispersion of continuous and discontinu- ous (stripe array) one dimensional gold grating struc-}

\section{tures}

Here we show the numerically calculated (COMSOL) transmission dispersion of two gold grating structures. figure S8(c) on the left reproduces the dispersion of a stripe array (see schematic figure $\mathrm{S} 8(\mathrm{a})$ ), as presented in the main manuscript in figure 2(b). On the right, in figure S8(d), we show the dispersion calculated for a similar grating, but with the addition of a $30 \mathrm{~nm}$ continuous gold layer (see schematic figure S8(b)). Although the strength of the modes as apparent in these transmission data are - as expected - very different, the position and shape of the grating scattered modes is very similar.

(a)

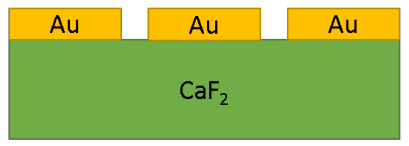

(c)

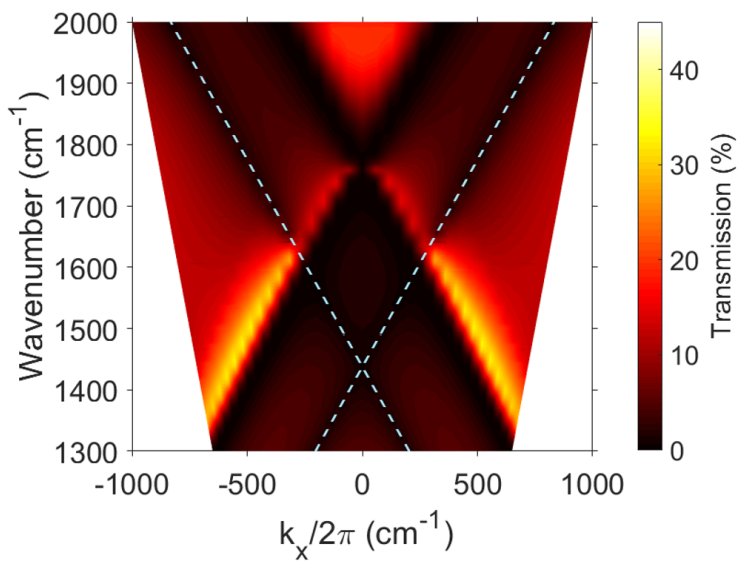

(b)

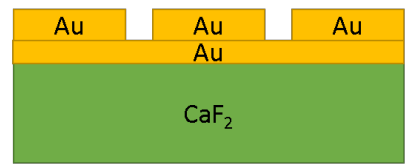

(d)

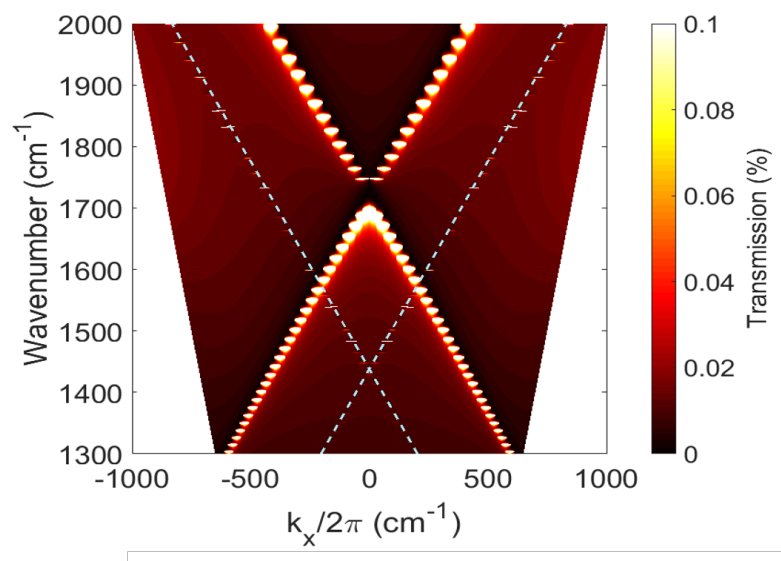

Figure S8: Schematics of: (a) discontinuous 1D metal grating on top of a $\mathrm{CaF}_{2}$ substrate, (b) continuous 1D metal grating on top of a $\mathrm{CaF}_{2}$ substrate. Dispersion plots calculated using COMSOL: (c) discontinuous 1D grating, (d) discontinuous 1D grating. For both dispersion plots the vibrational resonance was turned off (for clarity) by setting the oscillator strength to zero. The scattered air-light lines are shown as light-blue dashed lines. 


\section{Dispersion of DBR cavity modes for zero oscillator strength}

Here we show the dispersion of modes for the for the DBR cavity in the absence of the vibrational resonance. This was achieved by setting the oscillator strength to zero. These data should be compared to those in the main manuscript (figure 5) which were calculated in the presence of the vibrational resonance.

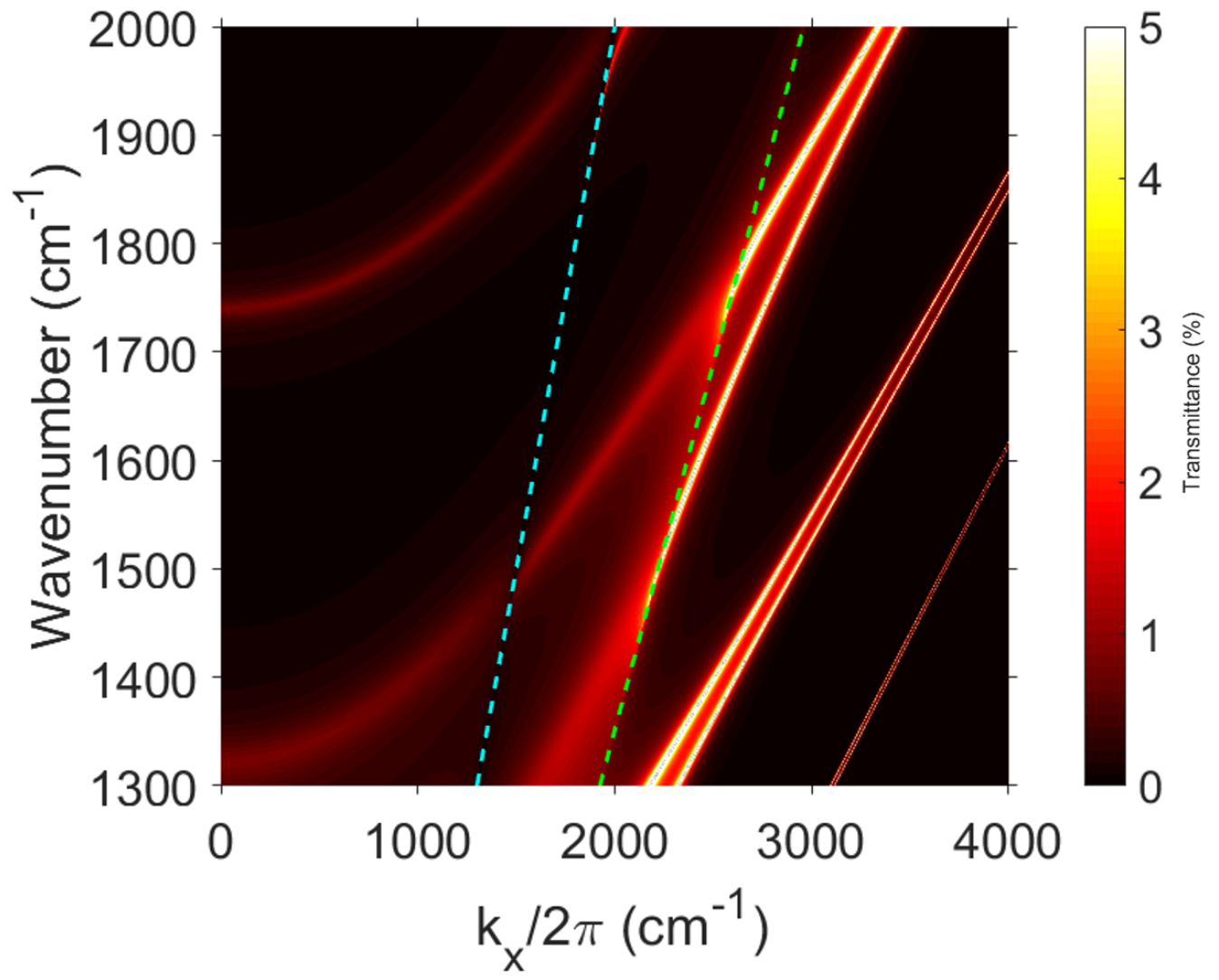

Figure S9: DBR-based cavity Dispersion plot based on Fresnel-type calculations. The absolute value of the TM polarised Fresnel coefficient is shown as a function of frequency (wavenumber) and in-plane wavevector. The blue and green dashed lines represent the air and PMMA light lines respectively.The oscillation strength of the molecular resonance was kept zero. Layer thicknesses and material parameters for the DBR mirrors are given in the methods section. 


\section{$7 \quad$ Line spectra}

Here we show the mode splitting which by looking at the line-spectra extracted form the experimental data shown in figure $2 \mathrm{c}$ and $2 \mathrm{f}$ of the main manuscript. For both of the linespectra shown the molecular $(\mathrm{C}=\mathrm{O})$ vibrational resonance is seen as a minimum at 1732 $\mathrm{cm}^{-1}$, as expected. For hybridisation with the plasmon mode of the single gold grating (black curve) the lower and upper polaritons are seen as minima at $\sim 1610 \mathrm{~cm}^{-1}$ and $\sim 1790$ $\mathrm{cm}^{-1}$ respectively. For hybridisation with the coupled plasmon mode of the cavity structure the lower and upper polaritons are seen as minima at $\sim 1590 \mathrm{~cm}^{-1}$ and $\sim 1760 \mathrm{~cm}^{-1}$ respectively. These line-spectra show that in both cases the splitting significantly exceeds the mode widths, see main text. 


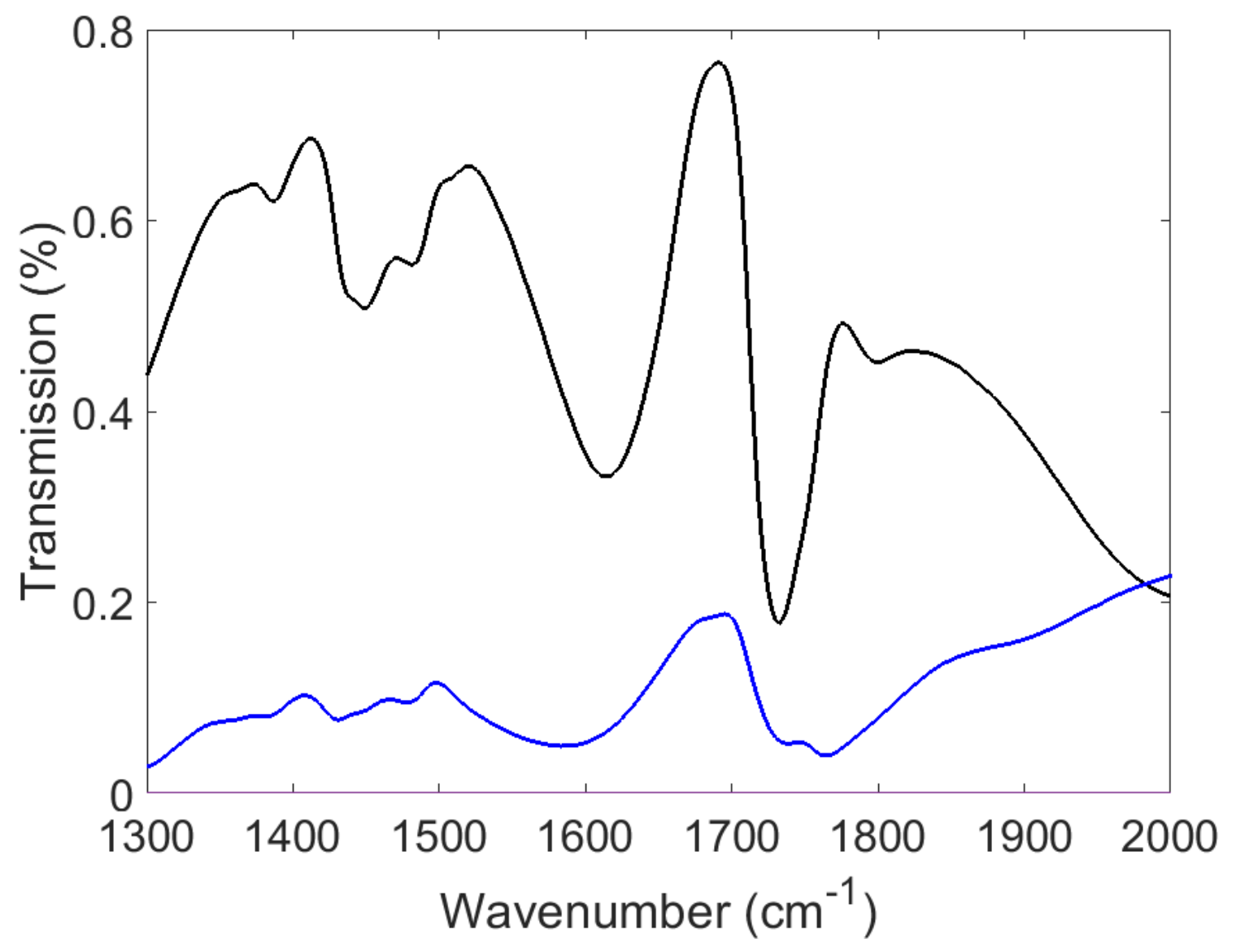

Figure S10: Line spectra. The line spectra for the FTIR intensity vs frequency for a $k_{x}$ value of $k_{x} \sim 170 \mathrm{~cm}^{-1}$ for the right hand panel of figure 2(c) is shown with solid black line and for figure $2(\mathrm{f})$ is shown with solid blue line. 


\section{Field profiles of the $\mathrm{TE}_{0}, \mathrm{TM}_{0}$ modes at higher $k_{x}$}

The data produced here are again from simulations using the Fresnel approach. We calculated the $T E_{0}$ and $T M_{0}$ field profiles at higher wavevector $\left(k_{x} \sim 1000 \mathrm{~cm}^{-1}\right)$. The field at lower wavevectors can be seen in Figure 4. Only relatively minor differences in the profiles (shapes) can be seen.
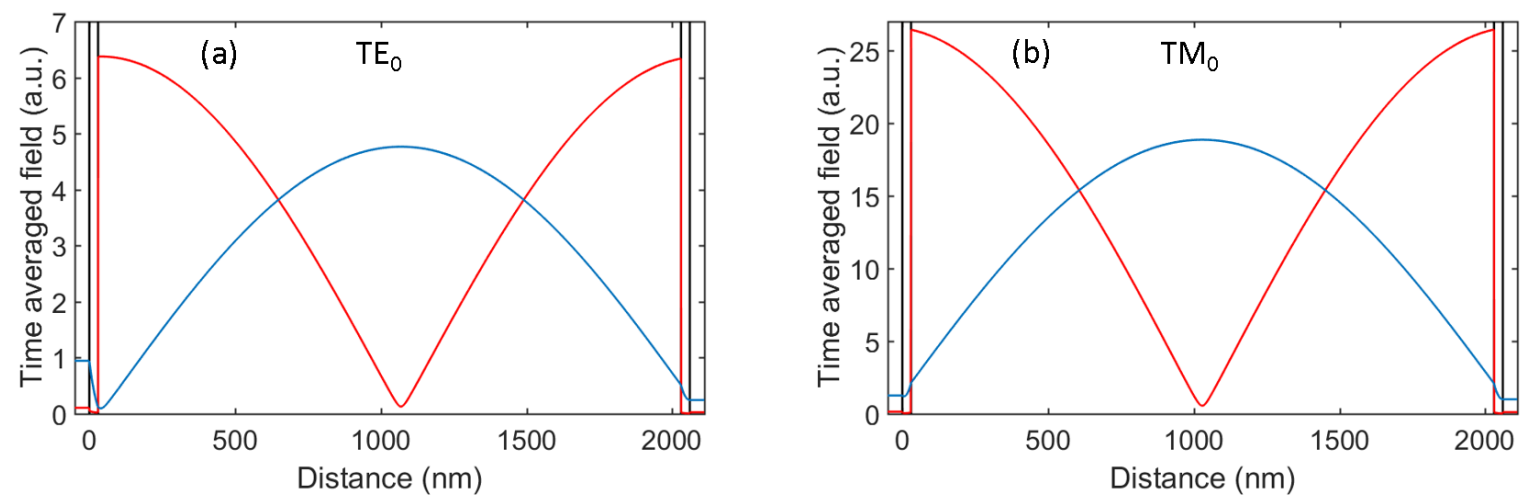

Figure S11: Field profile at higher $k_{x}$ Field profiles at $k_{x} \sim 1000 \mathrm{~cm}^{-1}$ were calculated. The field profiles of $T E_{0}$ and $T M_{0}$ were calculated at a frequency of $1732 \mathrm{~cm}^{-1}$. 


\section{Oscillator models for one molecular resonance and several photonic resonances}

There are two ways in which oscillator models can be calculated for one molecule and several photonic resonances. The first is a $(\mathrm{N}+1) \mathrm{x}(\mathrm{N}+1)$ oscillator model where $\mathrm{N}$ is the photonic resonances and 1 as single molecule resonance. The results obtained from solving for the eigenvalues and coefficients of equations 2 in the main manuscript are shown in figure S12a. The UP, MP and LP are shown in red, blue and pink respectively.

The second is a $2 \mathrm{Nx} 2 \mathrm{~N}$ oscillator model. Figure S12b shows the results obtained from solving for the eigenvalues and coefficients of equations 3 in the main manuscript. The UP and LP of $\mathrm{TM}_{0}$ modes are shown in red and blue. The UP and LP of $\mathrm{TM}_{-1}$ modes are shown in green and pink. Uncoupled $\mathrm{TM}_{0}, \mathrm{TM}_{-1}$ and molecular resonance modes are shown as dashed black lines.

(a)

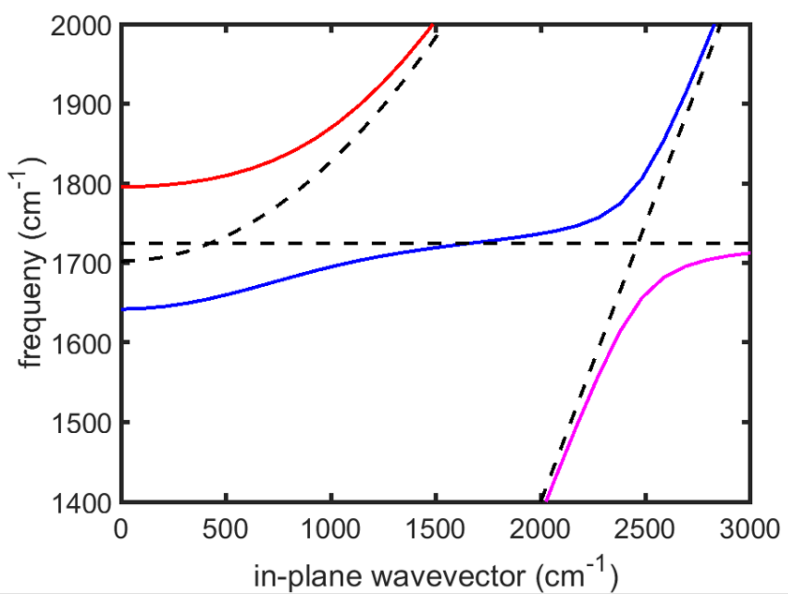

(b)

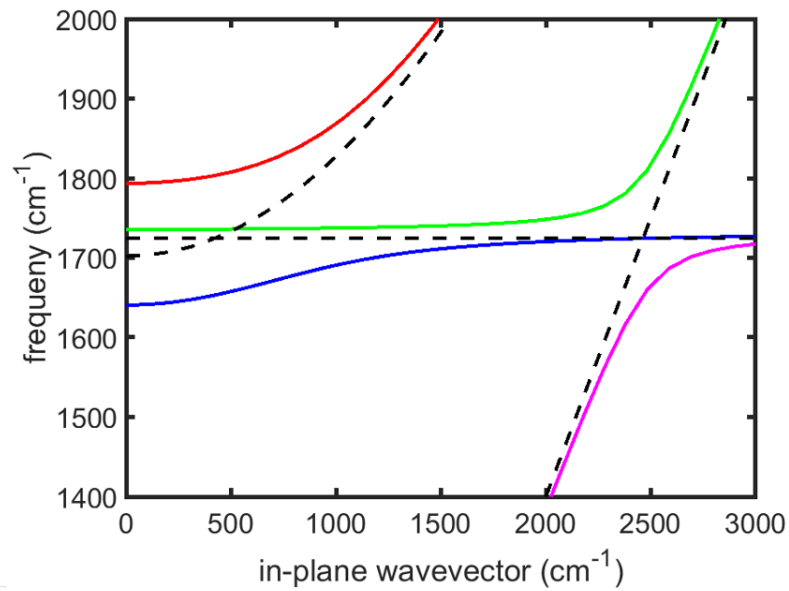

Figure S12: Different oscillator models (a) $(\mathrm{N}+1)$ oscillator model (b) $2 \mathrm{~N}$ oscillator model 